\title{
THE PSEUDO-DERIVATIVE OF A SUMMABLE FUNCTION.
}

BY PROFESSOR WILLIAM L. HART.

(Read before the American Mathematical Society September 8, 1920.)

Introduction.-In the present paper we shall consider two different types of derivative functions, to be termed pseudoderivatives. These derivatives will be defined for real-valued functions satisfying suitable conditions of summability. The definitions will state certain functional relations which the pseudo-derivatives must satisfy. These relations involve the well-known notion of convergence in the mean, explained in $\S 1$, below. The pseudo-derivative of the first type is considered in $\S 2$, and that of the second type in $\S 3$. The present paper is concerned merely with the definition of these derivatives and with a discussion of a few of their properties. Applications of such derivatives will be considered in a later paper.

All integrals in this paper are taken in the Lebesgue sense. A function $f$ will be termed integrable if both $f$ and $f^{2}$ are summable in the Lebesgue sense. All functions and variables will be supposed real-valued. The phrase almost everywhere will mean with the exception of at most a set of points of measure zero. In the discussion below two functions $F_{1}(x)$ and $F_{2}(x)$, defined on a set $E$, will be called the same if they are equal almost everywhere on $E$. In all equations below, as for example $F_{1}(x)=F_{2}(x)$, it will be understood that the equality may cease to hold on some sub-set of $E$ of measure zero.

1. Convergence in the Mean.-We shall have occasion to use a slight extension of the notion of convergence in the mean as it has been defined in the case of sequences of functions.* Let $q(s, h)$ be defined for $|h| \leqq h_{0}, h \neq 0$, and for $s$ on a measurable set $E$, where $s$ may be considered as a single or as an $m$-partite variable $\left(s_{1}, s_{2}, \cdots, s_{m}\right)$. For every $h$, suppose that $q(s, h)$ is integrable on $E$.

Definition 1. The function $q(s, h)$ converges in the mean

* Cf. Plancherel, Rendiconti del Circolo Matematico di Palermo, vol. 30 (1910), p. 292. 
to an integrable function $g(s)$ as $h$ approaches zero in case

$$
\lim _{h=0} \int_{E}[q(s, h)-g(s)]^{2} d s=0 .
$$

We shall say that $g(s)$ is the limit in the mean and shall write, for abbreviation,

$$
\operatorname{l.m}_{h=0} q(s, h)=g(s) .
$$

This type of convergence has properties similar to those* possessed by convergence in the mean as defined for sequences. Certain properties of $g(s)$ are listed below; the proofs of the statements will not be given because of their obvious relationship to proofs in the case of sequences of functions.

(a) A necessary and sufficient condition that (1) should hold for some function $g(s)$ is that

$$
\lim _{h_{1}, h_{2}=0} \int_{E}\left[q\left(s, h_{1}\right)-q\left(s, h_{2}\right)\right]^{2} d s=0 .
$$

(b) If $g(s)=g^{\prime}(s)$ and $g(s)=g^{\prime \prime}(s)$ both satisfy (2), then $g^{\prime}(s)=g^{\prime \prime}(s)$.

(c) At least one sequence of values $\left(h_{1}, h_{2}, \cdots, h_{n}, \cdots\right)$, with $\lim _{n=\infty} h_{n}=0$, can be selected so that $\lim _{n=\infty} q\left(s, h_{n}\right)=g(s)$, almost uniformly $\uparrow$ for $s$ on $E$.

$(d)$ The function $g(s)$ satisfies the equation

$$
\lim _{h=0} \int_{E} q^{2}(s, h) d s=\int_{E} g^{2}(s) d s .
$$

(e) Every sequence $\left(h_{1}, h_{2}, \cdots\right)$ with $\lim _{n=\infty} h_{n}=0$, corresponding to which the sequence $\left[q\left(s, h_{n}\right) ; n=1,2, \cdots\right]$ converges almost everywhere on $E$, has the property that

$$
\lim _{n=\infty} q\left(s, h_{n}\right)=g(s),
$$

almost everywhere on $E$.

2. The Pseudo-derivative of the First Kind.-Let $f(x)$ be defined and integrable for values of $x$ on some measurable set $E$ and let $q(x, h)=[f(x+h)-f(x)] / h$. For a given value of $h$, if $(x+h)$ is not in $E$, define $f(x+h)=f(x)$. If, for a given $h$ and $x, f(x+h)$ and $f(x)$ are infinities of the same sign, let

* Cf. Plancherel, loc. cit., p. 294.

$\dagger$ Cf. Plancherel, loc. cit., p. 292. 
$q(x, h)$ be given any arbitrary value, say zero. Then, for every $h \neq 0, q(x, h)$ is integrable on $E$.

Definition 2. The pseudo-derivative of $f(x)$ is $\mathrm{l}_{\mathrm{m}} \mathrm{m}_{\mathrm{h}=0}$ $q(x, h)$, provided this limit exists. It will be denoted by the symbol $f_{x}(x)$.

As a consequence of Definition $1, f_{x}(x)$ is unique if it exists, and satisfies the functional equation

$$
\lim _{h=0} \int_{E}\left[q(x, h)-f_{x}(x)\right]^{2} d x=0 .
$$

Moreover, at least one sequence $\left(h_{1}, h_{2}, \ldots\right)$ can be selected as in $(c), \S 1$, such that

$$
\lim _{n=\infty} q\left(x, h_{n}\right)=f_{x}(x),
$$

almost uniformly for $x$ on $E$. Suppose, for example, that $E$ is the set of all irrational numbers on the interval $(0,1)$ and let $f(x)=x$. Then it is easy to show that $f_{x}(x)=1$.

In analogy with the procedure followed in dealing with the classical derivative, let us denote the pseudo-derivative of order $n$ of $f(x)$ by the symbol $f_{x^{n}}(x)$, and let us define it to be the pseudo-derivative of $f_{x^{n-1}}(x)$, for $n=2,3, \cdots$. In the case of a function $f\left(x ; y_{1}, \cdots, y_{k}\right)$, let us define $f_{x}\left(x ; b_{1}, \cdots, b_{k}\right)$, the partial pseudo-derivative with respect to $x$ at the point $\left(y_{1}=b_{1}, \cdots, y_{k}=b_{k}\right)$, to be the pseudo-derivative in the sense of Definition 2 of the function $f\left(x ; b_{1}, \cdots, b_{k}\right)$.

In the two theorems which follow, conditions are given under which the pseudo-derivative is equal to the classical derivative.

TheOREm I. Suppose that the pseudo-derivative $f_{x}(x)$ exists and that $f(x)$ also possesses a derivative $d f(x) / d x$ almost everywhere on $E$. Then $d f(x) / d x=f_{x}(x)$ almost everywhere on $E$.

Since $d f(x) / d x$ exists, we have the equation

$$
\lim _{h=0} q(x, h)=\frac{d f(x)}{d x},
$$

almost everywhere on $E$. Since (5) and (6) both hold almost everywhere on $E$, it follows that the theorem is true.

Theorem II. Let the set $E$ be the closed interval $(a, b)$ and suppose that a constant $K>0$ exists such that, for all values of $h \neq 0$ and for all points $x$ on $(a, b),|q(x, h)| \leqq K$. Then, 
if the classical derivative exists at each point of $(a, b)$, the pseudoderivative $f_{x}(x)$ exists and $f_{x}(x)=d f(x) / d x$.

For values $x>b$ and $x<a$, define $f(x)$ by the equations

$$
\begin{aligned}
& f(x)=f(a)+(x-a) \frac{d f(a)}{d x} \quad(x<a), \\
& f(x)=f(b)+(x-b) \frac{d f(b)}{d x} \quad(x>b) .
\end{aligned}
$$

Since $f(x)$ is measurable, it is seen that $d f(x) / d x$ is also measurable. Moreover, as a consequence of a well-known property* of Lebesgue integrals it is allowable to invert $\lim _{h=0}$ and $\int_{(a b)}$ on the left side of the following equation and to obtain

$$
\lim _{h=0} \int_{(a b)}\left[q(x, h)-\frac{d f(x)}{d x}\right]^{2} d x=0 .
$$

Hence, by definition, $f_{x}(x)=d f(x) / d x$.

It is easily verified that Theorem II remains true if, in place of the assumption of the theorem in regard to $d f(x) / d x$, we assume that it exists everywhere on $(a, b)$ except at points $x$ on a set $H$ of measure zero.

3. The Pseudo-derivative of the Second Kind.-Let us consider a function $f(x, s)$ defined for values of the variable $x$ on a closed interval $(a, b)$ and for values of the $m$-partite variable $s=\left(s_{1}, \cdots, s_{m}\right)$ on a measurable set $E$. In all the discussion below suppose that, for every $x$ on $(a, b), f(x, s)$ is integrable on $E$ and let

$$
q(x, h, s)=\frac{f(x+h, s)-f(x, s)}{h} .
$$

For a given value of $h \neq 0$, if the point $(x+h)$ is not on $(a, b)$, define $f(x+h, s)=f(x, s)$. For points $s$ at which $f(x+h, s)$ and $f(x, s)$ are infinities of the same sign, let us give $q(x, h, s)$ any arbitrary value, say zero. Then, if $x=x_{0}$ is on $(a, b)$, it follows that the function $q\left(x_{0}, h, s\right)$, for every $h \neq 0$, is integrable on $E$.

Definition 3. The pseudo-derivative of the second kind of $f(x, s)$ with respect to $x$ at the point $x=x_{0}$, is $\operatorname{l.m}_{\cdot h=0} q\left(x_{0}, h, s\right)$, provided this limit exists. It will be denoted by the symbol $f_{x}^{\prime}(x, s)$.

* Cf. de la Vallée Poussin, Intégrales de Lebesgue, p. 44. 
For brevity, the designation "second kind" will be omitted in the future in this section of the paper in speaking of the pseudo-derivatives $f_{x}{ }^{\prime}(x, s)$. As a consequence of Definition 1 , if the function $f_{x}^{\prime}\left(x_{0}, s\right)$ exists, it is unique and satisfies the functional equation

$$
\lim _{h=0} \int_{E}\left[q\left(x_{0}, h, s\right)-f_{x}{ }^{\prime}\left(x_{0}, s\right)\right]^{2} d s=0 .
$$

If $f(x, s)$ is constant with respect to $s$ it is seen that $f_{x}{ }^{\prime}(x, s)$ reduces to the classical derivative of a function of the single variable $x$. Moreover, from $(c), \S 1$, it follows that at least one sequence $\left(h_{n} ; n=1,2, \cdots\right)$ can be selected with $\lim _{n=\infty} h_{n}=0$, such that

$$
\lim _{n=\infty} q\left(x_{0}, h_{n}, s\right)=f_{x}^{\prime}\left(x_{0}, s\right),
$$

almost uniformly for $s$ on $E$.

In the theorems that follow some useful properties of the pseudo-derivative $f_{x}{ }^{\prime}(x, s)$ are considered.

Theorem III. Suppose that at $x=x_{0}$ the pseudo-derivative $f_{x}{ }^{\prime}\left(x_{0}, s\right)$ exists, and that at $x=x_{0}$ the partial derivative (in the classical sense) $\partial f\left(x_{0}, s\right) / \partial x$ exists, finite or infinite, almost everywhere on $E$. Then it follows that $\partial f\left(x_{0}, s\right) / \partial x=f_{x}{ }^{\prime}\left(x_{0}, s\right)$.

By hypothesis we have, almost everywhere on $E$,

$$
\lim _{h=0} q\left(x_{0}, h, s\right)=\frac{\partial f\left(x_{0}, s\right)}{\partial x} .
$$

It is easily verified that the theorem is an immediate consequence of equations (9) and (10).

Theorem IV. Let the function $p(s)$ be bounded and measurable on $E$, and let $F(x, s)=f(x, s) p(s)$. Then, if $f_{x}^{\prime}\left(x_{0}, s\right)$ exists, it follows that there exists, likewise, $F_{x}{ }^{\prime}\left(x_{0}, s\right)=f_{x}^{\prime}\left(x_{0}, s\right) p(s)$.

The existence of $F_{x}{ }^{\prime}\left(x_{0}, s\right)$ follows from the fact that

$$
\lim _{h=0} \int_{E}\left[q\left(x_{0}, h, s\right) p(s)-f_{x}{ }^{\prime}\left(x_{0}, s\right) p(s)\right]^{2} d s=0,
$$

in case equation (8) holds.

Theorem V. If $f_{x}{ }^{\prime}\left(x_{0}, s\right)$ exists, and if $p(s)$ is any integrable function, the function $H(x)=\int_{E} f(x, s) p(s) d s$ has a derivative at the point $x=x_{0}$, given by the expression

$$
\frac{d H\left(x_{0}\right)}{d x}=\int_{E} f_{x}^{\prime}\left(x_{0}, s\right) p(s) d s .
$$


Let $\Delta H=\left[H\left(x_{0}+h\right)-H\left(x_{0}\right)\right]$. Then, because of the Schwarz inequality for integrals, it follows that

$$
\begin{array}{r}
\left|\frac{\Delta H}{h}-\int_{E} f_{x}{ }^{\prime}\left(x_{0}, s\right) p(s) d s\right|=\left|\int_{E}\left[f_{x}^{\prime}\left(x_{0}, s\right)-q\left(x_{0}, h, s\right)\right] p(s) d s\right| \\
\leqq \sqrt{\int_{E} p^{2}(s) d s} \sqrt{\int_{E}\left[f_{x}^{\prime}\left(x_{0}, s\right)-q\left(x_{0}, h, s\right)\right]^{2} d s,}
\end{array}
$$

and this expression, because of (8), approaches zero with $h$. This establishes the equation (11).

Let us represent by $M(x)$ the square root of the quantity $\int_{E} f^{2}(x, s) d s$. Then we have the following theorem.

Theorem VI. Suppose that, at $x=x_{0}, M\left(x_{0}\right) \neq 0$ and that there exists $f_{x}^{\prime}\left(x_{0}, s\right)$. Then, at $x=x_{0}, M(x)$ has the derivative

$$
\frac{d M\left(x_{0}\right)}{d x}=\frac{1}{M\left(x_{0}\right)} \int_{E} f_{x}{ }^{\prime}\left(x_{0}, s\right) f\left(x_{0}, s\right) d s .
$$

We shall establish the existence of $d M^{2}\left(x_{0}\right) / d x$; from this result (12) will follow immediately. We obtain, by use of (7),

$$
\begin{aligned}
& \frac{M^{2}\left(x_{0}+h\right)-M^{2}\left(x_{0}\right)}{h}=\int_{E} \frac{f^{2}\left(x_{0}+h, s\right)-f^{2}\left(x_{0}, s\right)}{h} d s \\
& =h \int_{E} q^{2}\left(x_{0}, h, s\right) d s+2 \int_{E} q\left(x_{0}, h, s\right) f\left(x_{0}, s\right) d s .
\end{aligned}
$$

As $h$ approaches zero, the first term on the right in (13) approaches zero by $(d), \S 1$. The second term approaches $2 \int_{E} f_{x}^{\prime}\left(x_{0}, s\right) f\left(x_{0}, s\right) d s$ because

$$
\begin{aligned}
\left|\int_{E}\left[q\left(x_{0}, h, s\right)-f_{x}{ }^{\prime}\left(x_{0}, s\right)\right] f\left(x_{0}, s\right) d s\right|^{2} & \\
& \leqq M^{2}\left(x_{0}\right) \int_{E}\left[q\left(x_{0}, h, s\right)-f_{x}{ }^{\prime}\left(x_{0}, s\right)\right]^{2} d s,
\end{aligned}
$$

which approaches zero with $h$. Consequently there exists

$$
\frac{d M^{2}\left(x_{0}\right)}{d x}=2 \int_{E} f_{x}{ }^{\prime}\left(x_{0}, s\right) f\left(x_{0}, s\right) d s .
$$

In the future suppose that $E$ is a closed interval $c \leqq s \leqq d$. Consider an infinite system of integrable functions

$$
I=\left[p_{n}(s) ; n=1,2, \cdots\right],
$$


which are unitary and orthogonal on $E$; that is,

$$
\int_{E} p_{i}(s) p_{j}(s)=d_{i j} \quad\left(d_{i i}=1, i=1,2, \cdots ; d_{i j}=0, i \neq j\right) .
$$

If $f(x, s)$ satisfies the conditions of this section of the paper, let

$$
z_{i}(x)=\int_{E} p_{i}(s) f(x, s) d s \quad(i=1,2, \cdots) .
$$

For brevity we shall term the $z_{i}(x)$ the Fourier coefficients of $f(x, s)$ with respect to the system $I$ and we shall call the vector $\xi(x)=\left[z_{1}(x), z_{2}(x), \cdots\right]$ the Fourier vector of $f(x, s)$. As an immediate consequence of Theorem $\mathrm{V}$ we may state that, if $f_{x}{ }^{\prime}\left(x_{0}, s\right)$ exists, the Fourier coefficients have derivatives at $x=x_{0}$ given by the expressions

$$
\frac{d z_{i}\left(x_{0}\right)}{d x}=\int_{E} p_{i}(s) f_{x}{ }^{\prime}\left(x_{0}, s\right) d s \quad(i=1,2, \cdots) .
$$

If $f_{x}^{\prime}(x, s)$ exists, let $\eta(x)=\left[y_{1}(x), y_{2}(x), \cdots\right]$ represent its Fourier vector with respect to the system $I$. It is seen from (16) that the $y_{i}$ and $z_{i}$ are related by the equation

$$
y_{i}(x)=d z_{i}(x) / d x .
$$

Let us suppose in the future that the system $I$ is complete for the class of all integrable functions on $E$. That is, we assume that there does not exist any integrable function $k(s) \neq 0$ such that $\int_{E} k(s) p_{i}(s)=0$ for $i=1,2, \cdots$. Then we shall establish the following theorem.

Theorem VII. For a value of $x$ at which $f_{x}{ }^{\prime}(x, s)$ exists,

$$
\lim _{h=0} \sum_{i=1}^{\infty}\left[\frac{z_{i}(x+h)-z_{i}(x)}{h}-y_{i}(x)\right]^{2}=0 .
$$

It has been proved by F. Riesz* that, if $g(s)$ and $h(s)$ are two integrable functions whose Fourier coefficients with respect to the system $I$ are $\left(a_{1}, a_{2}, \cdots\right)$ and $\left(b_{1}, b_{2}, \cdots\right)$ respectively, then

$$
\int_{E} g(s) h(s) d s=\sum_{i=1}^{\infty} a_{i} b_{i} .
$$

From this Riesz formula, it is easily verified that the infinite

* Cf. Plancherel, loc. cit., p. 296. 
sum in (17) is equal to

$$
\int_{E}\left[q(x, h, s)-f_{x}^{\prime}(x, s)\right]^{2} d s,
$$

so that Theorem VII is an immediate consequence of (8).

Before considering the next theorem, let us note some results which follow directly from the Riesz-Fischer* theorem concerning Fourier constants. If, for every $x$ on $(a, b), f_{x}{ }^{\prime}(x, s)$ exists and is zero almost everywhere on $E$, then, because of (16), the Fourier coefficients of $f(x, s)$ are constants. Consequently, by the Riesz-Fischer theorem, an integrable function $g(s)$ exists such that for every $x$ on $(a, b), f(x, s)=g(s)$. If $\xi(x)=\left[z_{1}(x), z_{2}(x), \cdots\right]$ is an infinite set of functions defined for $x$ on $(a, b)$ and is such that $\sum_{i=1}^{\infty} z_{i}{ }^{2}(x)$ converges for all values of $x$, then there exists, for every $x$ on $(a, b)$, a unique function $w(x, s)$ with the following properties:

(a) $w(x, s)$ is integrable on $E$.

(b) $\xi(x)$ is the Fourier vector of $w(x, s)$ with respect to $I$. Let $\eta(x)=\left[y_{1}(x), y_{2}(x), \cdots\right]$ be a second set of functions with the same properties as $\xi(x)$, and let $u(x, s)$ be the function associated with $\eta(x)$ and having the characteristics corresponding to $(a)$ and $(b)$ above. Then, in regard to $\xi(x)$ and $\eta(x)$, we may state the following theorem.

Theorem VIII. If, for a certain $x^{\prime}$ on $(a, b), \xi\left(x^{\prime}\right)$ and $\eta\left(x^{\prime}\right)$ satisfy (17), the function $w(x, s)$ has a pseudo-derivative at $x=x^{\prime}$ satisfying the equation $w_{x}^{\prime}\left(x^{\prime}, s\right)=u\left(x^{\prime}, s\right)$.

To establish the theorem consider the expression

$$
\int_{E}\left[\frac{w\left(x^{\prime}+h, s\right)-w\left(x^{\prime}, s\right)}{h}-u\left(x^{\prime}, s\right)\right]^{2} d s .
$$

By the Riesz formula, expression (18) is seen to equal the infinite sum entering in (17) with $x=x^{\prime}$. Hence (18) approaches zero with $h$ and, by definition, $w_{x}^{\prime}\left(x^{\prime}, s\right)=u\left(x^{\prime}, s\right)$.

Let us now establish an analog of the mean value theorem, for functions possessing pseudo-derivatives.

Theorem IX. For every $x$ on $(a, b)$ suppose that $f_{x}{ }^{\prime}(x, s)$ exists and that

$$
\lim _{x^{\prime}=x} \int_{E}\left[f_{x}^{\prime}\left(x^{\prime}, s\right)-f_{x}^{\prime}(x, s)\right]^{2} d s=0 .
$$

* Cf. Plancherel, loc. cit., p. 296. 
Let us assume, also, that $f_{x}{ }^{\prime}(x, s)$ is integrable with respect to $(x, s)$ in the rectangle $(a \leqq x \leqq b, c \leqq s \leqq d)$. Then it follows that, for all points $\left(x, x^{\prime}\right)$ on $(\bar{a}, b)$,

(20) $f\left(x^{\prime}, s\right)-f(x, s)=\left(x^{\prime}-x\right) \int_{0}^{1} f_{x}^{\prime}\left[x+u\left(x^{\prime}-x\right), s\right] d u$.

In (20), it should be recalled that, for given values of $\left(x, x^{\prime}\right)$, the conventions of this paper permit that the equation should not hold for a set of points $s$ with measure zero.

Let $\xi(x)$ be the Fourier vector of $f(x, s)$ relative to the complete system $I$. Then it is seen that the derivatives (16) exist and, moreover, that

$$
\frac{d z_{i}(x)}{d x}-\frac{d z_{i}\left(x^{\prime}\right)}{d x}=\int_{E}\left[f_{x}^{\prime}(x, s)-f_{x}^{\prime}\left(x^{\prime}, s\right)\right] p_{i}(s) d s .
$$

On applying the Schwarz inequality for integrals to the right side of (21), it is seen by (19) that the functions $d z_{i}(x) / d x$ are continuous for every value of $x$ on $(a, b)$. Therefore

$$
\begin{aligned}
z_{i}\left(x^{\prime}\right) & -z_{i}(x)=\left(x^{\prime}-x\right) \int_{0}^{1} \frac{d z_{i}\left[x+u\left(x^{\prime}-x\right)\right]}{d x} d u \\
= & \left(x^{\prime}-x\right) \int_{0}^{1}\left[\int_{E} p_{i}(s) f_{x}^{\prime}\left[x+u\left(x^{\prime}-x\right), s\right] d s\right] d u,
\end{aligned}
$$

where the last reduction was accomplished with the aid of (16). Let $\left(x^{\prime}, x\right)$ be fixed and let us show that the left and the right sides of (20) have the same Fourier coefficients. From this result, as an immediate consequence of the Riesz-Fischer theorem, it will follow that equation (20) is satisfied.

Let $K\left(x, x^{\prime}, s\right)$ represent the right of (20). From the theory of Lebesgue integrals* it follows that $K\left(x, x^{\prime}, s\right)$ is defined for all values of $s$ on $E$ except possibly for a set $E_{1}$ of measure zero. If arbitrary values are given to $K\left(x, x^{\prime}, s\right)$ at points in $E_{1}, K$ becomes an integrable function on the interval $E$. Let $\left(a_{1}, a_{2}, \ldots\right)$ be the Fourier coefficients of $K\left(x, x^{\prime}, s\right)$ with respect to $I$. Then we obtain

$$
\begin{array}{rlr}
a_{i} & =\left(x^{\prime}-x\right) \int_{E} p_{i}(s)\left[\int_{0}^{1} f_{x}^{\prime}\left[x+u\left(x^{\prime}-x\right), s\right] d u\right] d s \\
& =z_{i}\left(x^{\prime}\right)-z_{i}(x) & (i=1,2, \cdots),
\end{array}
$$

* Cf. de la Vallée Poussin, loc. cit., p. 53. 
where the last reduction was made by interchanging integrations in (23) and by comparing the result with (22). The interchange was permissible* because of our present hypotheses. Since $\left[z_{i}\left(x^{\prime}\right)-z_{i}(x)\right]$ is the $i$ th Fourier coefficient of the left side of (20), we have completed the proof of the theorem.

In a later paper the author will consider applications of the present results in the theory of functionals whose arguments are summable functions.

UNIVERSity OF Minnesota, December 15, 1920.

\title{
NOTE ON MINIMAL VARIETIES IN HYPERSPACE.
}

\author{
BY PROFESSOR C. L. E. MOORE.
}

(Read before the American Mathematical Society December 29, 1920.)

1. IT is known that a necessary and sufficient condition that a surface of two dimensions in hyperspace be minimal is the vanishing of the vector mean curvature. $\dagger$ It is the purpose of this note to show that mean curvature of a variety $V_{m}$ in a space of $n$ dimensions can be defined in the same way and that its vanishing is a necessary and sufficient condition that $V_{m}$ be minimal. I shall use the absolute calculus, since one of its chief merits is the ease with which invariants can be written down. In fact the very form of an expression shows whether or not it is invariant. Enough vector analysis is used to simplify the form of the expressions.

The variety $V_{m}$ can be written vectorially. in the form

Then

$$
y=y\left(x_{1}, x_{2}, \cdots, x_{m}\right) .
$$

$$
d s^{2}=d y \cdot d y=\sum_{1}^{m} a_{r s} d x_{r} d x_{s}
$$

If we write

$$
y_{r}=\frac{\partial y}{\partial x_{r}},
$$

* Cf. de la Vallée Poussin, loc. cit., p. 53.

†Wilson and Moore, "Differential geometry of two-dimensional surfaces in hyperspace," Proceedings of the Amer. Acad., vol. 52 (1916). 